\title{
Lower limb pulse rise time as a marker of peripheral arterial disease
}

\author{
Mikko Peltokangas, Damir Vakhitov, Velipekka Suominen, Janne Korhonen, Matti Huotari, Jarmo Verho, Juha \\ Röning, Ville M. Mattila, Pekka Romsi, Niku Oksala*, and Antti Vehkaoja*
}

\begin{abstract}
Objective: The aim of the study was to show if pulse rise times (PRTs) extracted from photoplethysmographic (PPG) pulse waves (PWs) have an association with peripheral arterial disease (PAD) or its endovascular treatment, percutanoeus transluminal angioplasty (PTA) of the superficial femoral artery. Methods: Lower and upper limb PPG PWs were recorded and analyzed from 24 patients who suffered from PAD. The measurements were conducted before and after the treatment, and one month later by using transmission-mode PPG-probes placed in the index finger and second toe. Ankle-to-brachial pressure index (ABI) and toe pressures were used as references in clinical patient measurements. PRTs, i.e. the time from the foot point to the peak point of the PW, were extracted from the PWs and compared bilaterally. The results from the PADpatients were also compared with 31 same-aged and 34 younger control subjects. Results: Statistically significant differences were found between the pre-treatment PRTs of the treated limb of the PAD-patients and the same-aged control subjects $\left(p<10^{-9}\right.$, Mann-Whitney U-test). The changes in the PRT of the treated lower limb were observed immeadiately after the PTA $(p<0.001$, Student's t-test), and after one month $(p<0.0005)$, whereas the PRTs of the non-treated lower limb and upper limb did not indicate changes between different examinations. Conclusion: Results show that a PRT greater than $240 \mathrm{~ms}$ indicates PADlesions in the lower limb. Significance: This proof-of-concept study suggests that the PRT could be an effective and easyto-use indicator for PAD and monitoring the effectiveness of its treatment.
\end{abstract}

Index Terms-Atherosclerosis, Photoplethysmography, Peripheral arterial disease, Pulse rise time, Pulse wave measurements.

\section{INTRODUCTION}

Peripheral arterial disease (PAD) is a specific form of atherosclerosis affecting mainly the lower limbs. It can be

Manuscript received on May 23, 2018, revised on August 27, 2018 and October 15,2018

M. Peltokangas, J. Verho, and A. Vehkaoja are with BioMediTech Institute and Faculty of Biomedical Sciences and Engineering, Tampere University of Technology, Tampere, Finland. Email addresses are firstname.lastname@tut.fi

D. Vakhitov and V. Suominen are with Division of Vascular Surgery, Department of Surgery, Tampere University Hospital, Tampere, Finland.

J. Korhonen is with Division of Interventional Radiology, Department of Radiology, Tampere University Hospital, Tampere, Finland

M. Huotari and J. Röning are with Infotech Oulu, Biomimetics and Intelligent Systems Group (BISG), University of Oulu, Oulu, Finland.

V. M. Mattila is with Department of Surgery, Tampere University Hospital, Tampere, Finland, and Surgery, Faculty of Medicine and Life Sciences, University of Tampere, Tampere, Finland.

P. Romsi is with Oulu University Hospital, Oulu, Finland

N. Oksala is with Division of Vascular Surgery, Department of Surgery, Tampere University Hospital, Tampere, Finland, and Finnish Cardiovascular Research Center Tampere, Surgery, Faculty of Medicine and Life Sciences, University of Tampere, Tampere, Finland.

*: N. Oksala and A. Vehkaoja contributed equally. either subclinical and asymptomatic or cause several symptoms such as intermittent claudication or in severe cases rest pain, which is typically a manifestation of critical limb ischemia. PAD is considered as a risk factor of acute cardiovascular events such as stroke or myocardial infarction and generally as an indicator of polyvascular disease, i.e. presence of atherosclerosis in two or more vascular beds. In addition to surgery, PAD is commonly treated by means of percutaneous transluminal angioplasty (PTA) which is a minimally invasive endovascular procedure for expanding the stenosed artery. The PTA initiates the revascularization which in most cases induces regenerative histological changes in the arteries. The patency rates after the femoropopliteal PTA are reported to vary from $75 \%-97 \%$ and $60 \%-84 \%$ for one- and two-year follow-up times [1], respectively. In order to ensure the technical success of the PTA, the patients are typically reexamined with the measurement of ankle-to-brachial pressure index (ABI) after one-month follow-up period.

The early diagnosis of the PAD is challenging due to its subclinical course. The measurement of ABI is considered as a gold standard even though its performance is challenged by diabetes and mediasclerosis [2]-[4] and even though it has found to have varying sensitivity and specificity [5]. Imaging based angiograms provide more precise information on the PAD lesions, but they are not suitable for screening studies because of their high costs, nephrotoxicity of the contrast agents and radiation exposure.

Photoplethysmography (PPG) is an optical and non-invasive technique for measuring the peripheral blood volume changes. A PPG probe consists of a light source and a photodetector. The light source illuminates the tissue, and the intensity of the reflected or transmitted light is observed by the photodetector. The intensity of the reflected or transimitted light depends on the optical properties of the tissue, including the amount of blood. It provides information on multiple biomedical parameters, such as heart rate, blood oxygen saturation, respiratory rate, and the condition of the vasculature [6], and could also provide a non-invasive and simple alternative for finding the PAD lesions. Earlier studies [6]-[11] have shown that different kinds of PPG-based lower limb pulse transit times (PTTs) are affected mainly by aging and the existence of PAD, but also by systolic blood pressure and height. Two of the major contributors, aging and PAD, affect the PTT in different directions: aging decreases the PTT but the existence of PAD lesions increases it [12]. Pulse rise time (PRT) is a time difference between two reference points found from the individual PPG PWs, i.e. peak and foot points. Allen et 
al. have reported differences in lower limb PPG-based PRTs between healthy subjects and PAD patients [6], [8], [9], but the authors are not aware of any prior study reporting the effects of PTA-treatment to PRT. In this proof-of-concept study, we investigate how the photoplethysmographic PRT changes as a result of the PTA procedure and if the PRTs could be a useful parameter to monitor patients' peri- and post-treatment response to PTA. We also study if the PRTs of the lower limb in patients with symptomatic PAD differ from the PRTs in the contralateral lower limb and from the lower limb PRTs of healthy subjects. We hypothesize that the stenoses in the arterial pathway increase the flow resistance, causing a low-pass filter-type response and delay in the PRT, whereas a successful PTA of the SFA decreases the resistance and changes the PRT towards healthier level.

\section{MATERIALS AND METHODS}

\section{A. Study subjects}

Altogether 89 Caucasian subjects were examined for the study. The patient measurements were conducted in two clinical studies (S1 and S2). The pulse wave (PW) signals of study S1 were recorded from 27 patients undergoing PTA for the superficial femoral artery (SFA) in 2016-2017. Three of them were excluded for the following reasons: research personnel unavailable at the follow-up visit, a patient was found to be unsuitable for PTA during the imaging and extremely low peripheral perfusion preventing the computation of PPG-based parameters. In study S1, 14 left and 12 right lower limbs were treated, including 2 patients with bilateral treatment. More detailed data on the study subjects is shown in Table I. The majority of the PAD patients suffered from intermittent claudication which is a typical symptom for PAD. In addition, most of the PAD patients had typical risk factors of atherosclerosis, such as dyslipidemia, diabetes, and arterial hypertension. Because almost all of the subjects having these diagnosed diseases had medication, the effects of the medication intended against atherosclerosis-promoting symptoms were not possible to analyze separately.

The inclusion criteria for study $\mathrm{S} 1$ were abnormal ABI reading, i.e. $\mathrm{ABI}<0.9$ or $\mathrm{ABI}>1.3$, relevant symptoms, stenosis in the SFA based on magnetic resonance angiography (MRA) and referral to the PTA of the SFA. The presence of a pacemaker and a possible risk that the study interferes the patient's treatment were considered as exclusion criteria. All patients except one met the criterion $\mathrm{ABI}<0.9$ in study $\mathrm{S} 1$, and only one of the included patients had a measured pre-treatment ABI higher than 1.3. Fifteen non-treated lower limbs out of $22(68 \%)$ had the ABI value outside the normal range at least in one of the pre- or post-treatment ABI measurements. More detailed distributions of the PAD patients' ABI readings are shown in Table II. Pre-treatment (measured during a visit at the outpatient clinic before the PTA) and post-treatment (followup visit) $\mathrm{ABI}$ and toe pressure measurements were routinely performed and used as references in the study.

Altogether 28 at least 65-year-old control subjects having normal ABI were examined in study $\mathrm{S} 2$ in 2015. In addition
Table I

DATA DESCRIBING THE STUDY SUBJECT POPULATION. THE VALUES ARE PRESENTED AS MEDIAN (1ST QUARTILE-3RD QUARTILE)

\begin{tabular}{|c|c|c|c|c|}
\hline Group & $\begin{array}{c}\text { Study S1 } \\
\text { PTA-patients } \\
(n=24)\end{array}$ & $\begin{array}{c}\text { Old } \\
(n=31)\end{array}$ & $\begin{array}{c}\text { Study S2 } \\
\text { Middle-aged } \\
\quad(n=15)\end{array}$ & $\begin{array}{l}\text { Young } \\
(n=19)\end{array}$ \\
\hline Age (years) & $72(68-76)$ & $71(67-77)$ & $58(54-62)$ & $28(25-33)$ \\
\hline Heart rate $(\mathrm{bpm})$ & & & & \\
\hline pre-PTA & $65.3(58.1-72.7)$ & 66.1 & 59.7 & 65.3 \\
\hline post-PTA & $64.2(58.1-72.5)$ & $(58.5-71.8)$ & $(55.1-65.2)$ & $(58.0-70.3)$ \\
\hline follow-up visit & $62.7(56.7-73.6)$ & & & \\
\hline \multicolumn{2}{|l|}{ Treated lower limbs: 26} & \multirow{8}{*}{$\begin{array}{c}1.10 \\
(1.06-1.22) \#\end{array}$} & \multirow{8}{*}{ \# } & \multirow{8}{*}{-} \\
\hline $\mathrm{ABI}$, pre-treatment & $0.61(0.50-0.75)$ & & & \\
\hline follow-up visit & $0.96(0.82-1.04)$ & & & \\
\hline change & $0.24(0.16-0.41)$ & & & \\
\hline Non-treated lower limbs: & 22 & & & \\
\hline ABI, pre-treatment & $0.88(0.75-1.02)$ & & & \\
\hline follow-up visit & $0.94(0.72-1.08)$ & & & \\
\hline change & $0.02(-0.07-0.25)$ & & & \\
\hline \multicolumn{2}{|c|}{ Toe pressure, treated limb: } & \multirow{8}{*}{ - } & \multirow{4}{*}{-} & \multirow{4}{*}{ - } \\
\hline pre-treatment $(\mathrm{mmHg})$ & $59(42-86)$ & & & \\
\hline follow-up visit $(\mathrm{mmHg})$ & $95(76-122)$ & & & \\
\hline change $(\mathrm{mmHg})$ & $22(7-41)$ & & & \\
\hline \multicolumn{2}{|c|}{ Toe pressure, non-treated limb: } & & \multirow{4}{*}{ - } & \multirow{4}{*}{ - } \\
\hline pre-treatment $(\mathrm{mmHg})$ & $76(53-118)$ & & & \\
\hline follow-up visit $(\mathrm{mmHg})$ & $100(66-122)$ & & & \\
\hline change $(\mathrm{mmHg})$ & $6(-24-24)$ & & & \\
\hline Men & $16(66.7 \%)$ & $12(38.7 \%)$ & $14(93.3 \%)$ & $18(94.7 \%)$ \\
\hline Diabetes & $11(45.8 \%)$ & $1(3.2 \%)$ & $1(6.7 \%)$ & $1(5.3 \%)$ \\
\hline Dyslipidemia & $22(91.7 \%)$ & $5(16.1 \%)$ & $0(0 \%)$ & $0(0 \%)$ \\
\hline History of smoking & $11(45.8 \%)$ & $7(22.6 \%)$ & $0(0 \%)$ & $0(0 \%)$ \\
\hline Rheumatoid arthritis & $3(12.5 \%)$ & $5(16.1 \%)$ & $0(0 \%)$ & $1(5.3 \%)$ \\
\hline Angina pectoris & $1(4.2 \%)$ & $1(3.2 \%)$ & $0(0 \%)$ & $0(0 \%)$ \\
\hline Critical limb ischemia & $4(16.7 \%)$ & $0(0 \%)$ & $0(0 \%)$ & $0(0 \%)$ \\
\hline Intermittent claudication & $22(91.7 \%)$ & $0(0 \%)$ & $0(0 \%)$ & $0(0 \%)$ \\
\hline Hemiplegia & $3(12.5 \%)$ & $0(0 \%)$ & $0(0 \%)$ & $0(0 \%)$ \\
\hline Hypertension & $19(79.2 \%)$ & $7(22.6 \%)$ & $0(0 \%)$ & $0(0 \%)$ \\
\hline Ischemic stroke & $1(4.2 \%)$ & $0(0 \%)$ & $0(0 \%)$ & $0(0 \%)$ \\
\hline Carotid stenosis & $2(8.3 \%)$ & $0(0 \%)$ & $0(0 \%)$ & $0(0 \%)$ \\
\hline Coronary artery disease & $7(29.2 \%)$ & $0(0 \%)$ & $0(0 \%)$ & $0(0 \%)$ \\
\hline Coronary thrombosis & $5(20.8 \%)$ & $0(0 \%)$ & $0(0 \%)$ & $0(0 \%)$ \\
\hline Cardiac failure & $5(20.8 \%)$ & $1(3.2 \%)$ & $0(0 \%)$ & $0(0 \%)$ \\
\hline Arrhytmia & $4(16.7 \%)$ & $2(6.5 \%)$ & $0(0 \%)$ & $0(0 \%)$ \\
\hline Renal failure & $4(16.7 \%)$ & $0(0 \%)$ & $0(0 \%)$ & $0(0 \%)$ \\
\hline
\end{tabular}

pre-treatment condition of treated limb, the follow-up visit condition of treated limb, the pre-treatment condition of non-treated limb, and the follow-up visit condition of treated limb (2-sided Mann-Whitney U-test).

Table II

DISTRIBUTIONS OF ABI READINGS FOR PAD PATIENTS.

\begin{tabular}{l|cc|cc}
\hline \hline & \multicolumn{2}{|c|}{ Treated limb } & \multicolumn{2}{c}{ Non-treated limb } \\
& pre-PTA & follow-up visit & pre-PTA & follow-up visit \\
\hline $\mathrm{ABI}<0.5$ & $5(19.2 \%)$ & $2(7.7 \%)$ & $1(4.6 \%)$ & $0(0.0 \%)$ \\
$\mathrm{ABI}<0.7$ & $16(69.6 \%)$ & $5(19.2 \%)$ & $3(13.6 \%)$ & $5(22.7 \%)$ \\
$\mathrm{ABI}<0.9$ & $24(92.3 \%)$ & $10(38.4 \%)$ & $12(54.6 \%)$ & $9(40.9 \%)$ \\
$0.9 \leq \mathrm{ABI} \leq 1.3$ & $1(3.9 \%)$ & $14(53.9 \%)$ & $9(40.9 \%)$ & $11(50.0 \%)$ \\
$\mathrm{ABI}>1.3$ & $1(3.9 \%)$ & $2(7.7 \%)$ & $1(4.6 \%)$ & $2(9.1 \%)$ \\
\hline \hline
\end{tabular}

to clinical patient measurements, 37 volunteer healthy 22-71year-old control subjects were examined in 2013-2017 in nonclinical environment in order to see if aging affects the PRTs. These measurements were conducted in Tampere University of Technology (21 subjects) and in University of Oulu (16 subjects). In these datasets, 3 subjects being at least 65 years of age were added and handled as a part of the clinical control subject group (old subjects). The rest of these 37 test subjects were divided into groups of 19 young (less than 40-year-old) and 15 middle-aged (40-64-year-old) subjects. 
a) Unprocessed lower limb PPG signals

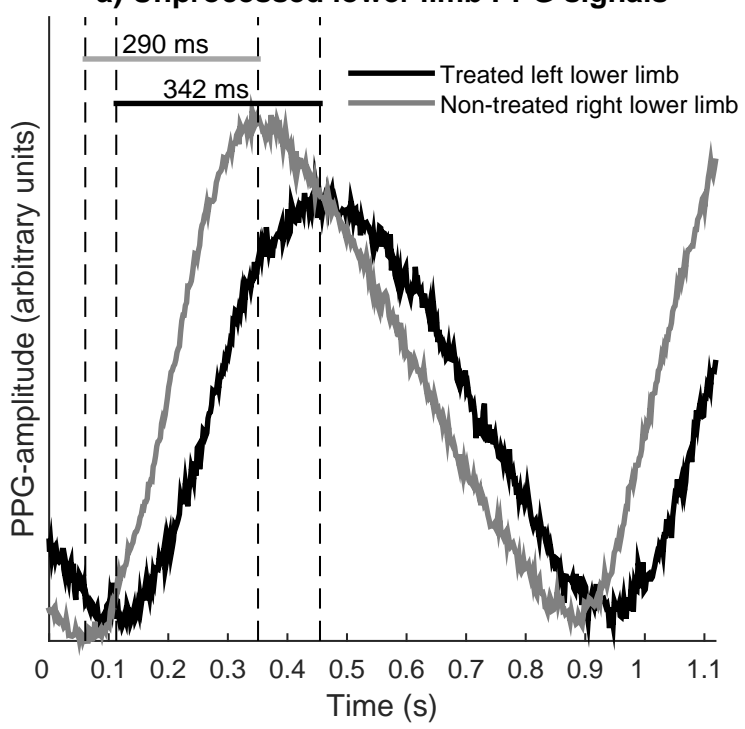

b) Filtered lower limb PPG signals

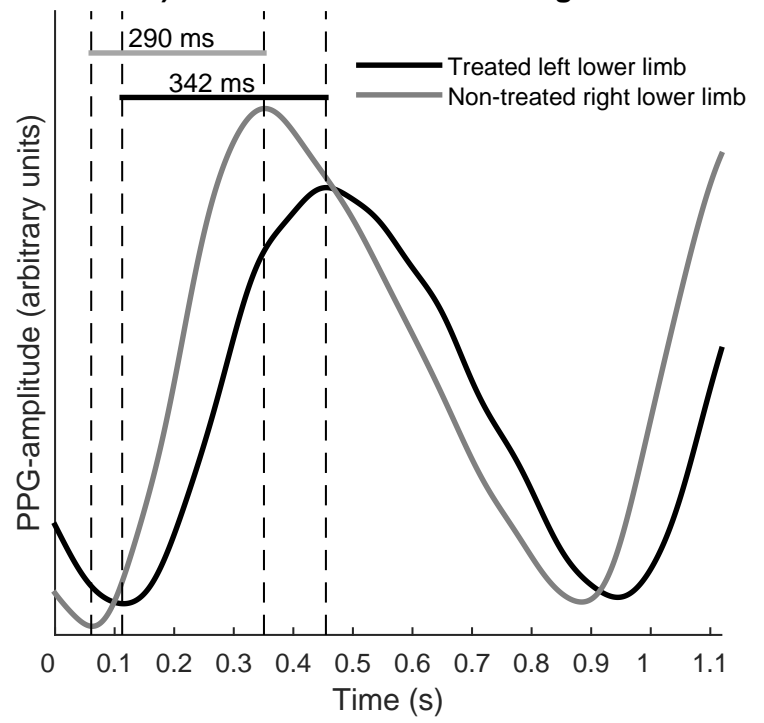

Figure 1. Examples of raw (a) and filtered (b) lower limb PWs as well as extracted PRTs for treated and non-treated lower limb before the PTA.

\section{B. Measurement hardware}

In studies $\mathrm{S} 1$ and $\mathrm{S} 2$ as well as in the measurements conducted in Tampere University of Technology, the measurement data was collected using transmission mode PPG-probes (S0010A, Shenzhen Med-link, China) having an excitation wave length of $905 \mathrm{~nm}$ connected into a synchronous wireless body sensor network (WBSN) presented in [13]. The passband of the PPG-amplifier of the WBSN was from $150 \mathrm{mHz}$ to $240 \mathrm{~Hz}$. A photoplethysmograph based on the phase-sensitive technique and transmission mode PPG-probes containing 940 $\mathrm{nm}$ light emitting diodes (LEDs) and photodiodes (BPW34, Vishay Semiconductors, USA) were used in the measurements conducted in University of Oulu [14]. The sampling frequencies were $500 \mathrm{~Hz}$ and $1000 \mathrm{~Hz}$ for WBSN and phase-sensitive PPG, respectively.

\section{Sensor placement}

With the PAD-patients, the PPG probes were placed on the index finger and both second toes for recording blood volume PW-signals. The test subjects were in supine position during the measurements. In study S1, the measurements were conducted during the pre-, peri-, and post-treatment phases of the PTA and during the follow-up visit after one month (median 33 days, IQR (30 days-36 days)). The sensors were placed before the normal preparation of the PTA and were removed within 5-10 minutes after the PTA. The duration of the measurement was approximately 15 minutes in study S2 and in the measurements conducted outside the hospitals.

\section{Pulse rise times}

PRT was defined as the time from the foot point to the peak point of the PW (Fig. 1). Before the determination of the foot and peak points, the PW-signals were lowpass-filtered with a finite impulse response filter having a cut-off-frequency of $10 \mathrm{~Hz}$, transition band of $10 \mathrm{~Hz}-12 \mathrm{~Hz}$, pass-band ripple of
$0.05 \mathrm{~dB}$, and a stop-band attenuation of $100 \mathrm{~dB}$ [15] using forward-backward filtering. After filtering, the end-point fixed linear trend was removed from each detected PW. Both filtered and raw signal examples on pre-treatment PWs are shown in Fig. 1 for treated and non-treated lower limb. The PAD-lesions are seen as attenuation of high-frequency features of PW, such as rounded sharp edges and a misssing dicrotic notch [8], [9], [11], [16]. As seen in Fig 1, the PAD-lesions may also change the morphology of the PW towards a sinusoidal wave and cause delayed arrival compared with a healthier contralateral limb [8], [9], [11].

The PRTs were computed as an average of at least one minute long recording. For the PAD patients, the lower and upper limb PRTs were extracted as immediate pre-treatment PRT ( $\left.t_{\text {before }}\right)$, immediate post-treatment PRT ( $\left.t_{\text {after }}\right)$, and PRT in the follow-up visit $\left(t_{\text {follow-up }}\right)$. The differences between different measurement events were computed between postand pre-treatment values as $t_{\mathrm{ab}}=t_{\mathrm{after}}-t_{\text {before }}$, between follow-up visit and pre-treatment values as $t_{\mathrm{fb}}=t_{\text {follow-up }}-$ $t_{\text {before }}$, and between follow-up visit and post-treatment values as $t_{\text {fa }}=t_{\text {follow-up }}-t_{\text {after }}$.

\section{E. Statistical methods}

Before comparing the results found for different study groups, the data normality was checked by using one-sample Kolmogorov-Smirnov test. Since all the rise time distributions were not normally distributed, non-parametric two-sided Mann-Whitney U-tests were implemented for comparing the control subjects' and the PAD patients' results, for comparing the PAD patients' results in different stages of operation, and for comparing the PAD patients' pre-treatment PRT distributions for treated and non-treated lower limbs. As all the differences between different measurement events (before the treatment, after the treatment, and follow-up visit) turned out to be normally distributed, their deviation from zero was tested by implementing Student's paired two-sided t-tests. The 
differences in the PRT values between different phases of the PTA treatment were not normally distributed, so nonparametric two-sided sign tests were implemented. P-values less than 0.05 were considered statistically significant. The performance of the PRTs in classifying the lower limbs into diseased or non-diseased was evaluated by means of receiver operating characteristic (ROC) curve analysis. In addition, the agreement between the ABI and PRT were evaluated by estimating Pearson's correlation coefficients for both absolute $\mathrm{ABI}$ and PRT as well as their change as a result of the PTA treatment.

Since vascular patients typically have multiple cardiovascular risk factors, multiple linear regression analysis was performed for both upper and lower limb PRTs of the PADpatients, and for the old, middle-aged and young control subjects in order to see which factors affect the PRTs. In order to avoid the distortion of the results caused by the risk factors being present only with few individual test subjects, only the predictors being present with at least 5 test subjects were taken into account. For the PAD-patients, the analysis was performed only for the pre-treatment values. Multiple linear regression analysis was implemented also for the changes in PRT having PTA and change in heart rate as predictors in order to check if the changes in the heart rate explain the changes in the PRT between a pre-treatment condition and a follow-up visit.

\section{F. Ethics and patient safety}

The patient measurements were approved by the local ethical review board of the hospital district (R14096 and R15107), and the Finnish National Supervisory Authority of Health and Welfare (Valvira, IDs 272 and 309). The study S1 was registered at ClinicalTrials.gov (ID: NCT02725307).

\section{RESULTS}

\section{A. Differences between study groups}

The distributions of the lower limb PRTs for different study groups are presented in Fig. 2. The pre-treatment PRTs of the treated lower limb were clearly greater than the PRTs of the lower limbs of the group of old control subjects $\left(p<10^{-9}\right)$ as presented in Fig 2. In addition, the differences in the lower limb PRT distributions between different-aged control groups were found to be small (Fig 2). The pre-treatment rise time distributions for treated and non-treated lower limbs were strongly overlapping, even though statistically signicant differences were found between them $(p \approx 0.017)$. At least a small difference was expected despite possible systemic effects of atherosclerosis since the pre-treatment ABI and toe pressure distributions were not equal (Table I). A small difference was also observed in the index finger PPG PRTs between the group of the old control subjects and the PAD-patients ( $p \approx 0.015$, Fig. 3 ) even though these distribtions were also strongly overlapping. In the index finger PRTs, the differences between the youngest group and older control groups were greater than in case of lower limb PRTs.

The ROC curves are shown in Fig. 4 in order to demonstrate the classification performance of the PRT. They are drawn

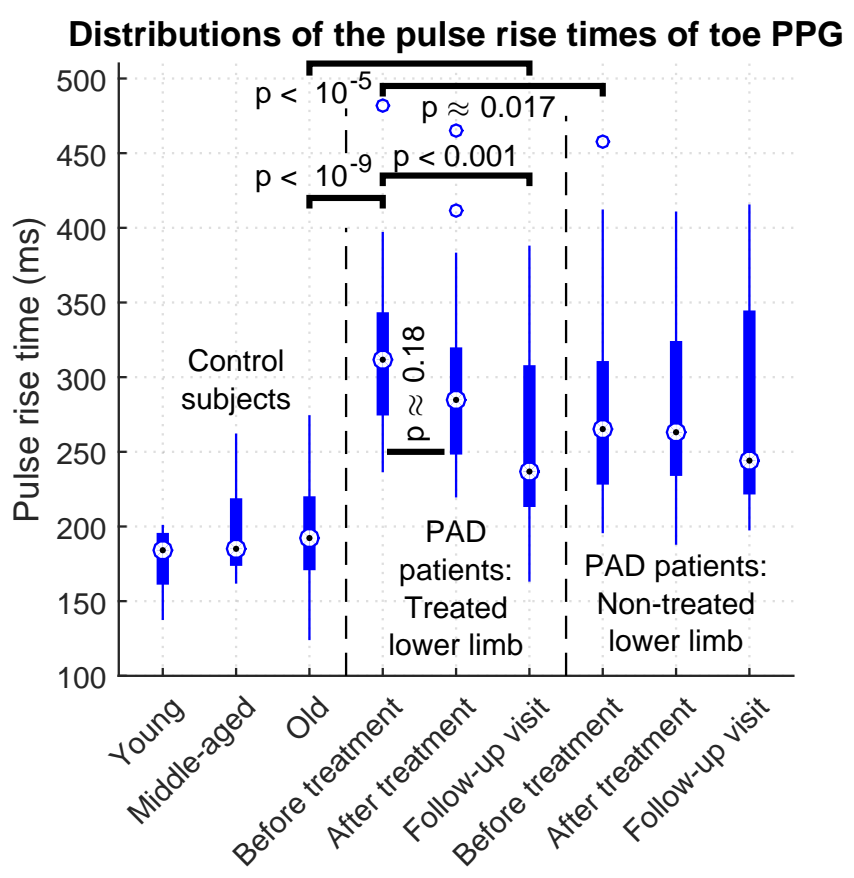

Figure 2. Distributions of the lower limb PRTs for different subject groups and treatment phases. The p-values are for two-sided Mann-Whitney U-test.

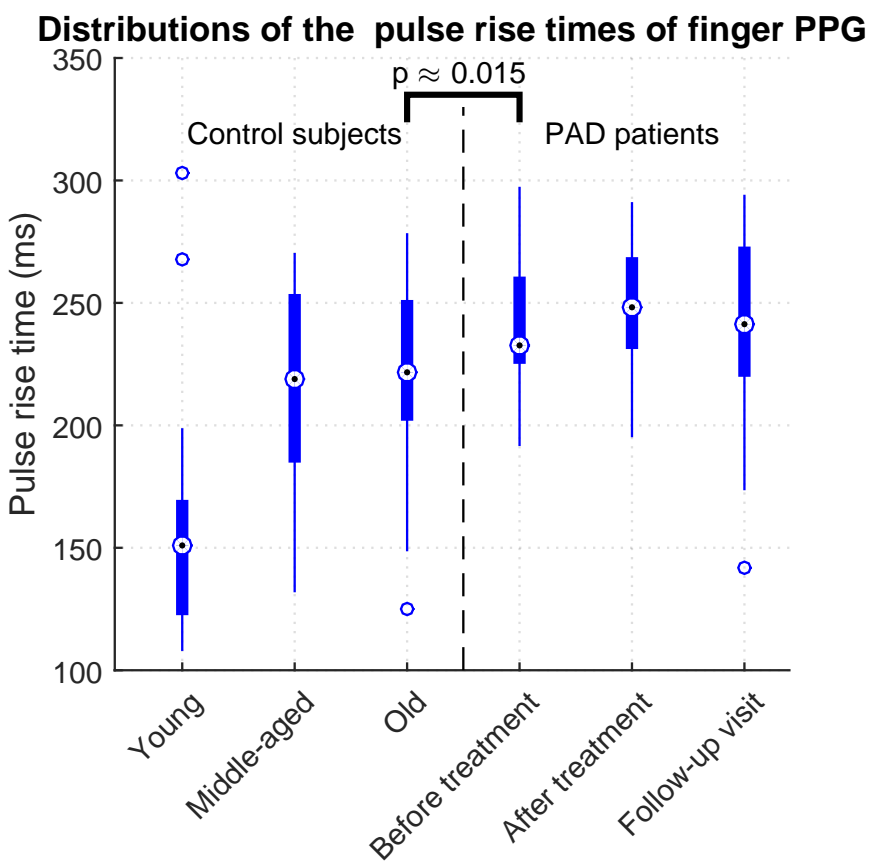

Figure 3. Distributions of the upper limb (index finger) PRTs for different subject groups and treatment phases. The p-value is for two-sided MannWhitney U-test.

for two different situations: 1) the treated limb of the PADpatients before the PTA vs. old healthy controls, and 2) the treated lower limb before the PTA vs. the non-treated lower limb before the PTA. The areas under curve (AUC) of 0.986 and 0.706 were obtained for the two situations, respectively. Even though the non-treated lower limb is less symptomatic than the contralateral limb, it does not mean that it does not 


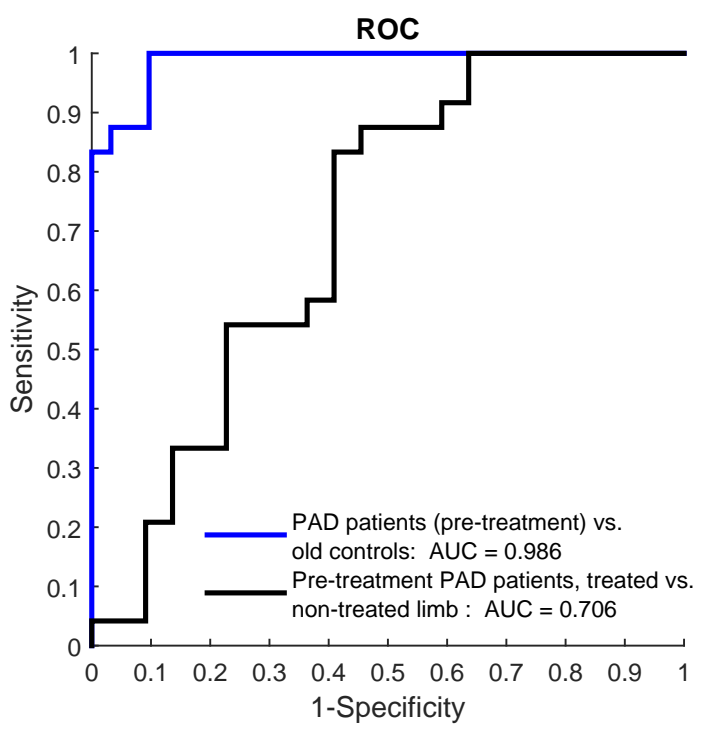

Figure 4. Receiver operating characteristic (ROC) curves for two situations: 1) the treated limb of PAD patients before the PTA vs. old healthy controls, and 2) treated lower limb before the PTA vs. non-treated lower limb before the PTA.

have any atherosclerotic PAD lesions due to the systemic nature of atherosclerosis. This is seen as the relatively low AUC for the distinction of treated and non-treated limb based on PRT. The partition values of $250 \mathrm{~ms}$ and $232 \mathrm{~ms}$ provide the sensitivities of 0.875 and 1.00 with specificities of 0.967 and 0.903 , respectively, for the comparison between healthy old controls and PAD patients. Thus, according to our results based on Caucasian subjects, the lower limb PRT greater than approximately $240 \mathrm{~ms}$ is a sign of possbile PAD lesions in that lower limb.

\section{B. Dependence between $A B I$ and PRT}

$\mathrm{ABI}$ and PRT values in different study groups and PAD patients at different phases are shown as a scatter plot in Fig. 5. Also the regression lines and Pearson's correlation coefficients are shown in Fig. 5 based on four different combinations of source data. A linear dependence between ABI and PRT cannot be assumed for ABI-values higher than 1.3 which indicates incompressible arteries. The normal range for ABI is $0.9-1.3$, so the ABI-values higher than 1.3 were excluded.

A dataset consisting of the old control subjects and the pre-treatment data of the PAD patients' treated limb yielded a correlation of $-0.81(-0.89 \ldots-0.69(95 \%$ confidence interval), $p<10^{-11}$ ). This evaluates how the PRT correlates with ABI in non-treated subjects and suggests that the PRT could discriminate at least the most extreme PAD lesions from healthy subjects. A correlation of $-0.64(-0.78 \ldots-0.42, p<$ $10^{-5}$ ) was found for a dataset consisting of PAD patients' pretreatment and follow-up visit results, and this describes how the ABI and PRT correlate in a data containing the treatment response. To estimate the overall dependence between the ABI and PRT, a correlation of $-0.58\left(-0.70 \ldots-0.43, p<10^{-9}\right)$ was found for the dataset consisting all the datapoints in Fig. 5. The least dependence $(r=-0.29,-0.01 \ldots-0.53, p<0.05)$

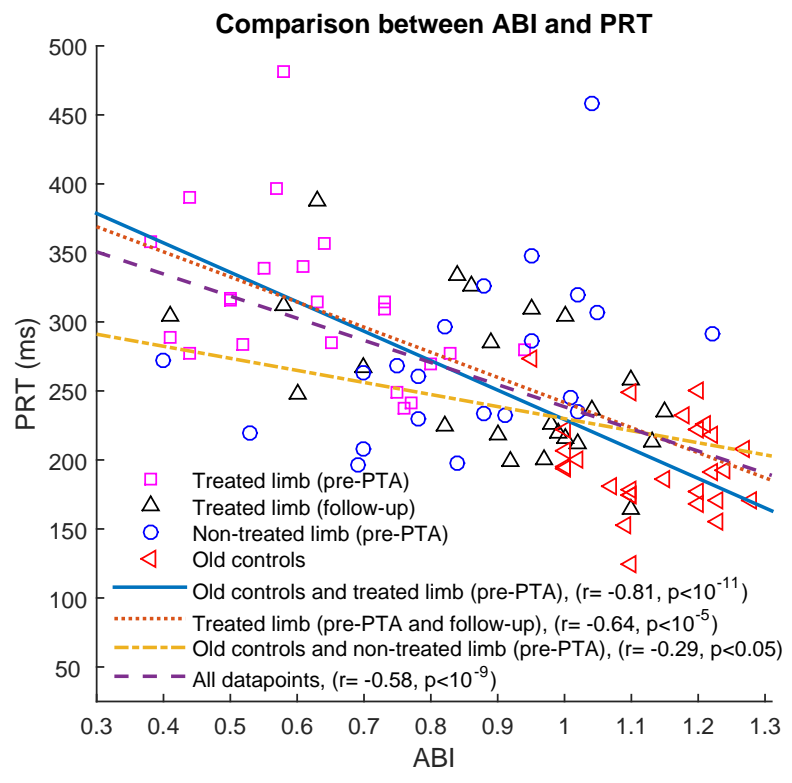

Figure 5. Comparison between ABI and PRT, grouped by different study groups. Also the regression lines based on different data are shown with Pearson's correlation coefficients $(r)$ and p-values.

between the ABI and PRT was observed in a dataset consisting the old control subjects and the pre-treatment data from the non-treated limb. In this dataset, the majority of the ABIreadings was in a narrower range than in the other datasets which may explain the poorer dependence.

\section{Changes caused by PTA}

Fig. 2 shows that the PRTs at pre-treatment, immediate posttreatment, and follow-up visit conditions of the treated limb are strongly overlapping, and there are even no statistically significant difference between the pre-treament and immediate post-treatment conditions $(p \approx 0.18)$. This is because the average or median change in PRT is relatively small with respect to its large between-subject variance.

However, at the individual level, there are group-wide consistent decreases in the PRTs of the treated limb as a result of the PTA. Clear differences are observed by using paired 2sided Student's t-tests for testing if the normally distributed differences between different conditions deviate from zero. Fig. 6 shows the distributions for paired changes between different situations, i.e. 1) immediately after and before the PTA, 2) follow-up visit and before the PTA, and 3) followup visit and immediately after the PTA. The differences are shown for both treated and non-treated lower limb as well as for the upper limb (index finger). The results in Fig. 6 show statistically significant differences in the treated lower limb: 1) $-33.9 \mathrm{~ms}(-74.6 \mathrm{~ms} \ldots-4.2 \mathrm{~ms})$ (median $(25 \% \ldots 75 \%$ quartiles)), $p<0.0005,2)-64.7 \mathrm{~ms}(-137.3 \mathrm{~ms} . .-20.1$ $\mathrm{ms}), p<0.0005$, and 3) $-59.0 \mathrm{~ms}(-110.5 \mathrm{~ms} \ldots-15.6 \mathrm{~ms})$, $p<0.001$ for the changes between immediate pre- and posttreatment, pre-treatment and follow-up visit, and immediate post-treatment and follow-up visit conditions, respectively. The signals recorded from non-treated lower limb and index finger 


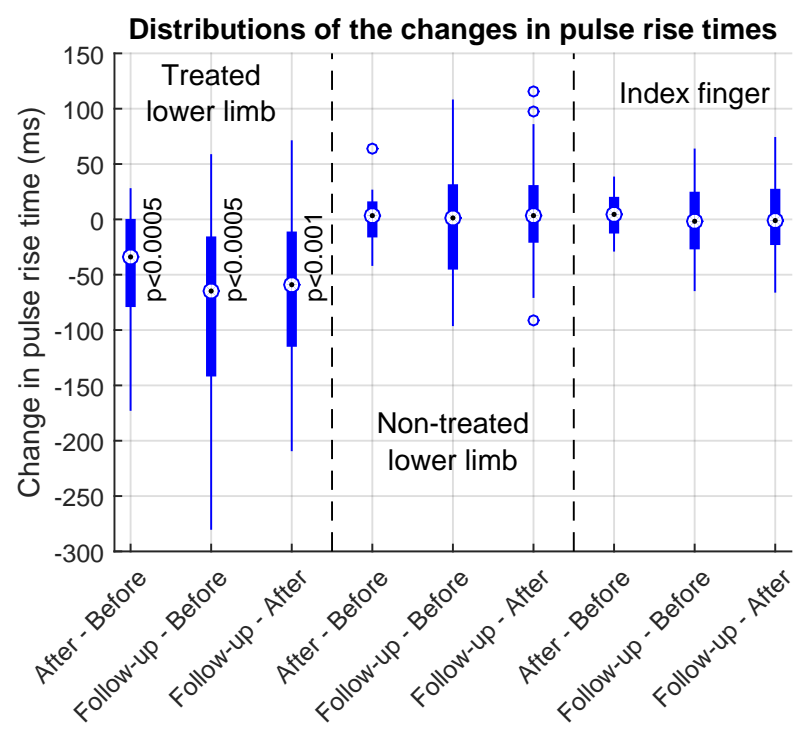

Figure 6. Changes in the rise times of the treated lower limb indicate statistically significant differences between different treatment phases whereas the changes in non-treated lower limb and index finger PPG do not show statistically significant differences between different measurement events.

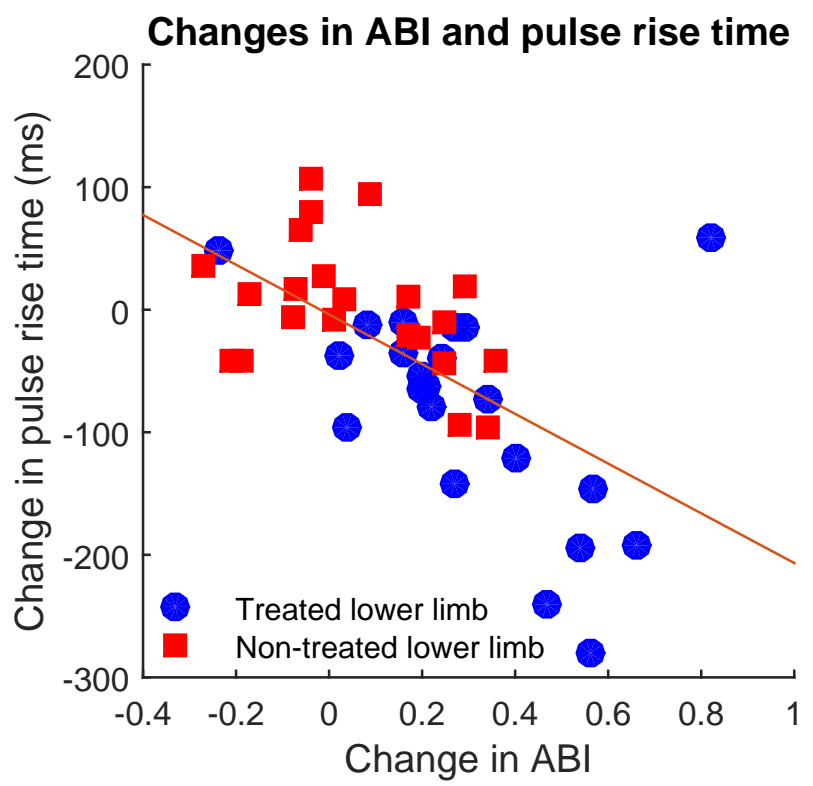

Figure 7. Changes in a lower limb PRT as a function of changes in the ABI.

do not show consistent or significant differences in either direction. The results in Fig. 6 show that the PTA causes immediate changes in PRTs in the treated limb and the changes continue during the follow-up period. The continuation of the change is most probably due to histological remodeling of the vasculature in the treated lower limb initiated by the revascularization. This is supported by the lack of consistent changes in the non-treated lower limb and in the index finger PRTs. Even though the PRTs of the treated lower limb change towards healthier direction, differences still remain between the old control subjects and the follow-up visit condition of the treated limb (Fig. 2, $p<10^{-5}$ ).

Changes in the PRT as a function of changes in the ABI are shown in Fig. 7 for both treated and non-treated lower limbs. Pearson's correlation coefficient between the change in lower limb PRT and ABI is $r=-0.595\left(p<5 \cdot 10^{-5}, 95 \%\right.$ confidence interval $-0.758 \ldots-0.362)$. The Pearson's linear correlation between the PRT and the other reference parameter, toe pressure, was less significant $r=-0.348(p=0.026$, $95 \%$-confidence interval $-0.592 \ldots-0.046$ ) which was the case also with the correlation between the reference parameters (ABI and toe pressure, $r=0.327, p=0.033,95 \%$-confidence interval $0.033 \ldots 0.568)$.

The inserted instrumentation did not cause significant changes: the median (interquartile range) changes in PRT values were $6.9 \mathrm{~ms}(-3.4 \mathrm{~ms}-17.6 \mathrm{~ms}, p \approx 0.08)$ and $6.0 \mathrm{~ms}(-12.2 \mathrm{~ms}-1.6 \mathrm{~ms}, p \approx 0.42)$ for 1$)$ pre-treatment situation vs. all the endovascular instruments inserted in the SFA, and 2) immediately after the last balloon angioplasty all the endovascular instruments in the SFA vs. immediate posttreatment condition, respectively.

\section{Multiple linear regression}

For the upper limb PRTs, the age, heart rate and smoking are statistically significant predictors. With lower limb PRTs, the PAD is a clearly more significant predictor than age, heart rate, other cardiovascular disorders or symptoms concomitantly present in the PAD-patients. The visually observed stronger age dependence of the upper limb (Fig. 3) in comparison with the lower limb (Fig. 2) PRT supports the results of the multiple linear regression analysis. Table IV shows the results of multiple linear regression analysis for the changes in PRT due to PTA and change in heart rate. This shows that the change in heart rate does not explain the changes between the pre-treatment state and the follow-up visit.

\section{DISCUSSION}

The results show that the lower limb PRT increases with the PAD lesions compared with healthy subjects or contralateral

Table III

MULTIPLE LINEAR REGRESSION COEFFICIENTS $(\beta)$ WITH P-VALUES FOR UPPER AND LOWER LIMB PRTS. STATISTICALLY SIGNIFICANT $(p<0.05)$ VALUES ARE HIGHLIGHTED.

\begin{tabular}{lcccc}
\hline \hline & \multicolumn{2}{c}{ Lower limb } & \multicolumn{2}{c}{ Upper limb } \\
& $\beta$ & $p$ & $\beta$ & $p$ \\
\hline (Intercept, ms) & 244 & $1.7 \cdot 10^{-7}$ & 173 & $1.3 \cdot 10^{-8}$ \\
Age (ms/year) & 0.63 & 0.015 & 1.4 & $5.7 \cdot 10^{-9}$ \\
Heart rate (ms/bpm) & -1.3 & 0.002 & -1.1 & 0.005 \\
PAD (ms) & 42 & $4.2 \cdot 10^{-4}$ & 16 & 0.513 \\
Diabetes (ms) & 15 & 0.256 & -11 & 0.440 \\
Dyslipidemia (ms) & 47 & 0.006 & 0.93 & 0.947 \\
Smoking (ms) & 1.0 & 0.917 & 22 & 0.018 \\
Rheumatoid arthritis (ms) & -11 & 0.513 & 24 & 0.050 \\
Intermittent claudication (ms) & 46 & 0.002 & 4.0 & 0.855 \\
Hypertension (ms) & -44 & 0.003 & 12 & 0.343 \\
Coronary artery disease (ms) & -14 & 0.556 & 11 & 0.642 \\
Coronary thrombosis (ms) & -18 & 0.510 & -34 & 0.273 \\
Cardiac failure (ms) & 7.0 & 0.720 & -16 & 0.412 \\
Arrhythmia (ms) & 45 & 0.101 & 25 & 0.337 \\
\hline F-statistics vs. constant model & $5.3 \cdot 10^{-17}$ & & $7.4 \cdot 10^{-11}$ \\
\hline \hline
\end{tabular}

$\mathrm{PAD}=$ peripheral arterial disease 
Table IV

MULTIPLE LINEAR REGRESSION COEFFICIENTS $(\beta)$ WITH P-VALUES BETWEEN PRE-TREATMENT CONDITION AND FOLLOW-UP VISIT FOR THE CHANGES IN UPPER LIMB PRTS WITH CHANGE IN HEART RATE (HR) AND PTA AS PREDICTORS. STATISTICALLY SIGNIFICANT $(p<0.05)$ VALUES ARE HIGHLIGHTED.

\begin{tabular}{lcc}
\hline \hline & $\beta$ & $p$ \\
\hline (Intercept, ms) & -2.9 & 0.73 \\
Change in HR (ms/bpm) & -0.94 & 0.29 \\
PTA (ms) & -55.4 & $3.3 \cdot 10^{-05}$ \\
\hline F-statistics vs. constant model & $9.9 \cdot 10^{-5}$ \\
\hline \hline
\end{tabular}

healthier lower limb. The differences between the contralateral lower limbs in the PAD-patients are clearly smaller than between the limb with PAD lesions and healthy same-aged control subjects. An obvious reason is that the PAD lesions are often bilaterally present. In this study, the ABI measurements of the non-treated lower limb provided conflicting readings in the two measurements: $68 \%$ of the PAD-patients had abnormal $\mathrm{ABI}$ also in the non-treated lower limb at least in one of the two ABI measurements (ABI before the PTA and during the follow-up visit) which supports our observation of bilaterality. This is a limitation of the study as the conclusions from the condition of the non-treated asymptomatic or healthier lower limb are not necessarily reliable due to the challenges with the current reference methods [4], [5]. However, the distributions of the ABI-readings of the treated and non-treated lower limbs were (Table I) more distinct than the corresponding distributions of the PRTs (Fig. 2) and the other reference value, toe pressure (Table I).

A notable issue at group-level is that the PRT differences between the follow-up visit condition and the old control subjects remain (Fig. 2), despite the PTA treatment and the resulting trend towards smaller PRTs. This difference is also seen in ABI-readings (Table I). The major reason most likely is in the systemic nature of atherosclerosis. In the PTA of the SFA, only the most stenosed or occluded segments of the SFA are dilated, and the possible lesions in the more distal segments of the lower limb arteries are left untreated.

The index finger PRTs do not change consistently between the different measurements within the PAD-patients. In general, the stenoses in the upper limbs are less common than in the lower limbs [17], [18]. The small differences observed in the index finger PRTs between the old control subjects and PAD-patients before the PTA are assumed to be caused by excessive arterial stiffening which is typical with the PADpatients having several cardiovascular risk factors. The stiffening of the arteries increases the pulse wave velocity which makes the heart-beat induced wave and first reflected wave more and more overlapping, causing delayed temporal location of the peak value compared with the healthy arteries having more distinct waves. Differences in other upper limb PWderived features, such as augmentation index and reflection index, between healthy controls and atherosclerotic patients have also been observed in an earlier study [19].

According to the presented results, the upper limb PRTs are mainly associated with the aging $\left(1.4 \mathrm{~ms} /\right.$ year, $\left.p \approx 5.7 \cdot 10^{-9}\right)$ and history of smoking ( $22 \mathrm{~ms}, p \approx 0.018)$. Also the lower limb PRTs depend slightly on the age $(0.63 \mathrm{~ms} / \mathrm{year}$, $p \approx 0.015$ ), but PAD (42 ms, $p \approx 4.2 \cdot 10^{-4}$ ) and its typical symptoms, such as intermittent claudication $(46 \mathrm{~ms}$, $p \approx 0.002$ ) and dyslipidemia (47 ms, $p \approx 0.006$ ), were found to have a more significant effect. Based on the presented results, the lower limb PW-derived features are more sensitive than the upper limb PWs to reveal arterial diseases which can be clinically seen as a continuum of degenerative changes. One possible reason is that the higher hydrostatic pressure in the lower limbs at standing or sitting positions causes physiological thickening of the arterial walls, making the arteries of the lower limb more vulnerable to atherosclerotic changes [20]. On the other hand, the arterial pathway from the heart to the toe is longer than distance from the heart to the finger which may have an effect, too.

Earlier studies have found association between age and PTT [8]-[10] as well as association between the time delays of pulse arrivals between healthy and diseased lower limbs [11]. In both cases, peak or foot points of the PW are utilized as reference points. A recent study [12] suggests that the PTA affects positively to the lower limb PTT of a treated limb. There are also studies reporting differences in the PRTs between PADpatients who have abnormal ABI and healthy control subjects as well as differences between the contralateral lower limbs of the PAD-patients [7]-[9]. However, the authors are not aware of the prior studies reporting the changes of PRT caused by the PTA treatment. The present study shows that the PRTs decrease towards healthier values as a result of successful PTA. The positive changes found in the vascular dynamics as a result of the PTA are supported also by Yokoyama et al. [21] who have reported a decrease in brachial-ankle pulse wave velocity (baPWV) in diabetic patients having PAD compared with diabetic patients without PAD lesions even though baPWV normally increases with age and arterial stiffening. In their study, an increase in baPWV values of the diabetic patients was observed after the successful PTA. A positive change after the lower limb PTA in an upper limb derived vascular parameter, radial artery derived aortic augmentation index, has been reported as a result of beneficial systemic vascular effects after revascularization and remodeling [22]. However, the present study did not find consistent improvement in the upper limb PRT or non-treated lower limb PRT.

When considering the pressure as a driving force or input in the arterial system, arterial tree as a conduit system or a transfer function having a certain frequency response, and the observed peripheral blood volume pulse as an output or a response of the system, the increase in the flow resistance of the arterial pathway decreases the cut-off frequency of the arterial tree. This causes higher-frequency features to be attenuated which is seen as rounded features of the PW and the delays in the response of the system, i.e. the arrival of the peripheral peak blood volume in the case of PPG measurement. The dimininished arterial compliance, which is associated with aging, decreases the PTT by $0.58 \mathrm{~ms} /$ year ... $0.80 \mathrm{~ms} /$ year [7], [10], but PAD increases in the lower limb PRT and foot- and peak point-based PTTs with PAD-patients [8], [9], [12] which is in line with the present results and our assumption. With respect to the present and earlier results, the 
increase in flow resistance is a dominating factor compared with the decreases in the arterial compliance in PAD-patients.

Previous studies have reported varying sensitivity of $15 \%$ $79 \%$ and specificity of $83 \%-99 \%$ for a test of $\mathrm{ABI}<0.9$ [5], which is the current gold standard in daily clinical practice in screening and monitoring of the PAD. ABI has challenges especially in the patients having mediasclerotic changes. In such patients, normal or too high ABI readings are expected, despite the stenoses, since the stiffening of the lower limb arteries prevents the compression of the arteries with a cuff used in the ABI measurement. Lower limb PRT or other lower limb PW-derived markers [8], [9], [11], [16], [19] could play an additional diagnostic role along with conventional screening tools such as ABI and toe pressure measurement especially in patients having stenoses and mediasclerotic changes concomitantly present in the lower limbs. As the PRT is not significantly disturbed from the inserted endovascular instrumentation, it could be suitable even for peri-operative monitoring to check the patient's response to the treatment. However, the validation of the method requires further studies with higher sample size and especially diabetic patients having $\mathrm{ABI}>1.3$.

\section{CONCLUSIONS}

The present proof-of-concept study suggests that lower limb PRT is an effective marker of PAD lesions. Based on the data recorded from 89 Caucasian subjects, the lower limb PRT greater than approximately $240 \mathrm{~ms}$ indicates PAD lesions and the patients may benefit from additional vascular examinations. The age does not explain the difference, and the method is selective (AUC=0.986) for the PAD lesions in the lower limb: The study showed that there were immediate changes (-33.9 ms (-74.6 ms...-4.2 ms), $p<0.0005)$ of the lower limb PRTs in the limb that underwent PTA of the SFA, and the changes became more visible (-64.7 ms (-137.3 ms...$20.1 \mathrm{~ms}), p<0.0005)$ after one month follow-up period, most probably due to histological remodeling of the lower limb arteries after the revascularization. In the case of non-treated lower limb or upper limb, no consistent changes were observed in the PRT. Based on the present and previous studies, lower limb PW-derived features [8], [9], [12], [16], [19] could add to diagnostics especially in patients presenting with stenoses and mediasclerotic changes of the lower limb arteries. Despite the promising results, further studies with higher sample sizes should confirm and validate the results.

\section{ACKNOWLEDGMENT}

The authors would like to thank all the volunteer test subjects for their valuable contribution. The personnel in Tampere and Oulu University Hospitals (Department and Polyclinics of Vascular Surgery) are acknowledged for their contribution in collecting the reference values.

The work was funded by the Doctoral Programme of the President of Tampere University of Technology, Business Finland as a part of project VitalSens (decision ID 40103/14), Academy of Finland as part of project Vascular Biomechanics Assessment using printed soft electronics and advanced signal processing (VBA) (decisions 310617 and 310618) and grants from Finnish Cultural Foundation/Pirkanmaa Regional Fund, Tekniikan edistämissäätiö and Emil Aaltonen Foundation.

\section{REFERENCES}

[1] J. R. Laird, "Limitations of percutaneous transluminal angioplasty and stenting for the treatment of disease of the superficial femoral and popliteal arteries," Journal of Endovascular Therapy, vol. 13, no. 2_suppl, pp. II-30-II-40, 2006.

[2] V. Suominen et al., "Prevalence and risk factors of pad among patients with elevated abi," European Journal of Vascular and Endovascular Surgery, vol. 35, no. 6, pp. 709-714, 2008.

[3] S. Alatab et al., "Impact of hypertension on various markers of subclinical atherosclerosis in early type 2 diabetes," Journal of Diabetes \& Metabolic Disorders, vol. 13, no. 1, p. 1, 2014.

[4] M. T. Romanos, A. Raspovic, and B. M. Perrin, "The reliability of toe systolic pressure and the toe brachial index in patients with diabetes," Journal of foot and ankle research, vol. 3, no. 1, p. 31, 2010.

[5] D. Xu et al., "Sensitivity and specificity of the ankle-brachial index to diagnose peripheral artery disease: a structured review," Vascular Medicine, vol. 15, no. 5, pp. 361-369, 2010

[6] J. Allen, "Photoplethysmography and its application in clinical physiological measurement." Physiological measurement, vol. 28, no. 3, pp. R1-39, 2007.

[7] J. Allen and A. Murray, "Age-related changes in peripheral pulse timing characteristics at the ears, fingers and toes," Journal of human hypertension, vol. 16, no. 10, p. 711, 2002.

[8] J. Allen et al., "Photoplethysmography detection of lower limb peripheral arterial occlusive disease: a comparison of pulse timing, amplitude and shape characteristics," Physiological measurement, vol. 26, no. 5 , p. 811, 2005.

[9] J. Allen et al., "A prospective comparison of bilateral photoplethysmography versus the ankle-brachial pressure index for detecting and quantifying lower limb peripheral arterial disease," Journal of vascular surgery, vol. 47, no. 4, pp. 794-802, 2008.

[10] M. Nitzan, B. Khanokh, and Y. Slovik, "The difference in pulse transit time to the toe and finger measured by photoplethysmography," Physiological measurement, vol. 23, no. 1, p. 85, 2002.

[11] R. Erts et al., "Bilateral photoplethysmography studies of the leg arterial stenosis," Physiological Measurement, vol. 26, no. 5, p. 865, 2005.

[12] M. Peltokangas et al., "Effects of percutaneous transluminal angioplasty of superficial femoral artery on photoplethysmographic pulse transit times," IEEE Journal of Biomedical and Health Informatics, pp. 1-1, 2018.

[13] M. Peltokangas et al., "Monitoring arterial pulse waves with synchronous body sensor network," Biomedical and Health Informatics, IEEE Journal of, vol. 18, no. 6, pp. 1781-1787, Nov 2014.

[14] M. Huotari et al., "Photoplethysmography and its detailed pulse waveform analysis for arterial stiffness," Journal of Structural Mechanics, vol. 44, no. 4, pp. 345-362, 2011.

[15] S. C. Millasseau et al., "The vascular impact of aging and vasoactive drugs: comparison of two digital volume pulse measurements," American journal of hypertension, vol. 16, no. 6, pp. 467-472, 2003.

[16] M. Peltokangas et al., "The effect of percutaneous transluminal angioplasty of superficial femoral artery on pulse wave features," Computers in biology and medicine, vol. 96, pp. 274-282, May 2018.

[17] M. Bae et al., "Upper limb ischemia: clinical experiences of acute and chronic upper limb ischemia in a single center," The Korean journal of thoracic and cardiovascular surgery, vol. 48, no. 4, p. 246, 2015.

[18] W. J. McCarthy et al., "Result of bypass grafting for upper limb ischemia," Journal of Vascular Surgery, vol. 3, no. 5, pp. 741-746, 1986.

[19] M. Peltokangas et al., "Parameters extracted from arterial pulse waves as markers of atherosclerotic changes: Performance and repeatability," IEEE Journal of Biomedical and Health Informatics, vol. 22, no. 3, pp. 750-757, May 2018.

[20] M. Rac-Albu et al., "The role of ankle-brachial index for predicting peripheral arterial disease," Maedica, vol. 9, no. 3, p. 295, 2014.

[21] H. Yokoyama et al., "Pulse wave velocity in lower-limb arteries among diabetic patients with peripheral arterial disease," Journal of atherosclerosis and thrombosis, vol. 10, no. 4, pp. 253-258, 2003.

[22] V. Jacomella et al., "The impact of endovascular lower-limb revascularisation on the aortic augmentation index in patients with peripheral arterial disease," European Journal of Vascular and Endovascular Surgery, vol. 45, no. 5, pp. 497-501, 2013. 